\title{
Right ventricle myectomy
}

\author{
Konstantin V. Borisov ${ }^{1,2}$ \\ ${ }^{1}$ University Paris Descartes, Sorbonne Paris Cite, 75475 Paris, France; ${ }^{2}$ German-Russian Cardiac Clinic, Moscow, Russia \\ Correspondence to: Konstantin V. Borisov, MD. Slavyanckaya sq., 2/5/4 b3, Moscow, 109074, Russia. Email: kvborisov@inbox.ru.
}

\begin{abstract}
Right ventricular (RV) hypertrophy is common in patients with hypertrophic cardiomyopathy (HCM), and is associated with more severe disease. Conventional surgical strategies such as the traditional Morrow procedure pose a particularly high risk to patients with severe hypertrophy and RV obstruction, for whom the most appropriate therapeutic approach has not yet been established. We have proposed a new technique for surgical correction in patients with hypertrophic obstructive cardiomyopathy and severe hypertrophy, which involves approaching the area of obstruction by entering through the conal part of the RV. This novel technique provides effective elimination of biventricular obstruction and the precise removal of the areas of septal fibrosis in patients with hypertrophic obstructive cardiomyopathy. The current literature review analyzes the indications and various techniques for performing a RV myectomy, and presents the results of follow-up assessments in patients with biventricular obstruction and severe hypertrophy.
\end{abstract}

Keywords: Hypertrophic obstructive cardiomyopathy; biventricular obstruction; myectomy; right ventricular hypertrophy

Submitted Apr 19, 2017. Accepted for publication Jul 04, 2017.

doi: 10.21037/acs.2017.07.10

View this article at: http://dx.doi.org/10.21037/acs.2017.07.10

\section{Introduction}

Hypertrophic cardiomyopathy (HCM) is characterized by left and/or right ventricular (RV) hypertrophy, with predominant involvement of the interventricular septum (1). Substantial right ventricular outflow tract (RVOT) pressure gradients may also occur in HCM and are usually associated with subaortic gradients.

The surgical correction of ventricular outflow obstruction in patients with HCM is usually based on transaortic access to the left side of the IVS followed by the resection of the sub-aortic muscle, essentially as devised by Morrow (2). However, this approach is not applicable to patients with extreme left ventricular hypertrophy, biventricular obstruction, and RV obstruction. In the absence of accepted standards for surgical treatment of hypertrophic obstructive cardiomyopathy (HOCM) for patients with severe hypertrophy, we have decided to investigate a route distinct from the conventional Morrow procedure by approaching the hypertrophied interventricular septal region through the conal part of the RV and performing a partial excision of additional myocardium on the right side of the septum.

Conceptually, our approach (3) offers a number of advantages: it allows the excision of the asymmetrically hypertrophied area of the ventricular septum without penetration into the left ventricle cavity and avoids mechanical damage to the heart conduction system and aortic valve. For the surgeon, the approach improves visual inspection of the area to be resected. A comprehensive literature review was conducted to clarify the indications for RV myectomy, including indications for patients with biventricular obstruction and septal myocardial fibrosis. Analysis of surgical techniques for RV myectomy and their outcomes suggests that our approach may allow for more patient-specific treatment strategies.

\section{RV involvement in HCM}

The majority of young adults with asymmetric hypertrophy in Teare's original report (4) of sudden death had both right and left ventricular hypertrophy (Figure 1A). The hypertrophic process in HCM may also affect the RV $(5,6)$. 
Recent studies have shown that cardiac hypertrophy is not limited to the left ventricle. Magnetic resonance studies by Maron et al. (7) demonstrated that the RV wall thickness was increased in patients with HC compared to controls. In a third of patients with HCM, RV wall thickness and/or mass were increased, including about $10 \%$ with extreme RV wall hypertrophy (>10 mm). Most patients with HCM (53\%) had diffuse RV hypertrophy involving all three segments of the RV.

Current research demonstrates that RV wall thickening is common in this HCM population, and therefore the hypertrophic process in this disease process appears to be more diffuse than previously appreciated (7). RV hypertrophy assessed by two-dimensional echocardiography is common in patients with HCM. In these patients, McKenna et al. (5) demonstrated a significant association between RV involvement and severity of symptoms. These authors also reported an increased incidence of ventricular tachycardias and supraventricular arrhythmias in their patients. The presence of RV hypertrophy was associated with more severe disease.

The study of Pagourelias et al. (8) demonstrated the importance of $\mathrm{RV}$ diastolic function markers in the prognosis of HCM patients. The establishment of RV restrictive physiology appears to have significant predictive value in HCM, regardless of the presence of other risk factors. The main findings of a study by Finocchiaro et al. (9) suggested that $\mathrm{RV}$ dysfunction according to the $\mathrm{RV}$ myocardial performance index is common in patients with HCM and related to the degree of LV dysfunction. RV dysfunction was independently associated with an increased likelihood of death or transplantation. The results of Nagata et al. (10) suggest that HCM patients with RV hypertrophy on cardiovascular magnetic resonance (CMR) imaging have a greater incidence of cardiovascular events than patients without RV hypertrophy.

RVOT obstruction is seen in $15 \%$ of patients with $\mathrm{HCM}$ (11). A small RV cavity is an occasional finding $(6,12)$ and RVOT stenosis, frequently found, is usually caused by the bulging of the hypertrophied septum $(11,13)$.

\section{RV anatomy-visual inspection}

Maron et al. (14) assessed morphology of the RV of outflow tract anatomy in four patients at the time of right ventriculotomy and muscular resection (and at autopsy in two of these patients). RV anatomy was also assessed in the autopsy of another patient who underwent myotomy- myectomy without right ventriculotomy. In these direct visual inspections, greatly hypertrophied myocardium was observed, associated with reduced RV cavity size. In four patients, the crista supraventricularis muscle (including both the septal and parietal limbs) was enormously thickened, as was the moderator band and other RV trabeculae; the RV free wall was thickened in all patients.

Quintana et al. (15) noted that the presence of significant diffuse septal hypertrophy contributed to biventricular obstruction in their entire cohort of 11 patients. In these patients, there was a significant right-sided septal hypertrophy bulging into the RVOT as an isolated cause of obstruction, or in association with septal muscle bundles or free wall hypertrophy. In this series, all patients had concomitant RV and LV obstruction.

In our study (3), patients with simultaneous obstruction of the LV mid-cavity and RVOT exhibited RV obstruction due to excessive hypertrophy of the trabeculae, the IVS septum and in some cases of the RV anterior wall. Reduced size of the RV cavity was also evident in all of these patients. Another study, this time of 35 pediatric HOCM patients, demonstrated hypertrophied septomarginal trabeculae and $\mathrm{RV}$ wall thickening in all subjects following direct visual inspection of the RV anatomy (16). Maximum RV anterior wall thickness was increased in 33 (94\%) children. Eleven pediatric patients (31\%) demonstrated an extreme $\mathrm{RV}$ anterior wall hypertrophy $(>10 \mathrm{~mm})$. In all patients, RV hypertrophy was combined with massive hypertrophy of the IVS and left ventricular hypertrophy. These data suggest that HCM is generally a particularly severe process affecting both ventricles (7).

\section{RV myectomy-surgical techniques}

Limited data in the literature is available on treatment approaches to biventricular obstruction $(3,14,15)$. Harken described a technique for surgical treatment of hypertrophic subvalvular stenosis in 1964 (17). A transverse incision was made through the RV and a tremendous amount of material was excised. The incision was directed by a finger through the aorta into the left ventricle. The authors noted that if the left ventricle is accidentally incised, the septal defect produced can readily be repaired. A temporary complete heart block, however, occurred in this particular case, necessitating pacemaker implantation. Our technique of HOCM surgical treatment $(3,16)$ allows one to avoid damaging the cardiac conduction system and enables performance of the excision without penetration into the 
A

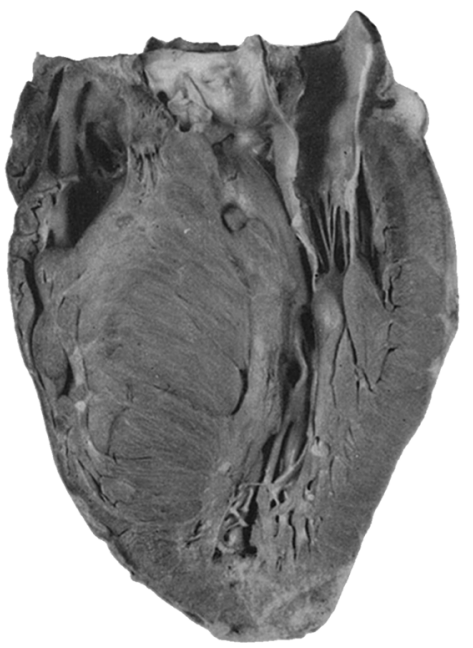

B

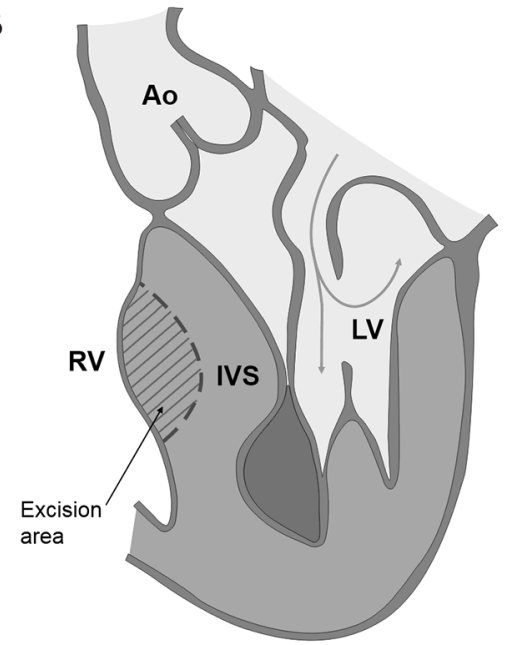

Figure 1 View of heart (a pathologic specimen) with severe hypertrophy (A) and surgical technique (B) for HOCM patients with severe hypertrophy and biventricular obstruction. (A) A localized and diffuse hypertrophy of the interventricular septum and RV involvement (4) (Copyrighted with permission of BMJ Publishing Group Ltd). (B) The excision of the hypertrophied area of the interventricular septum causing simultaneous obstruction of the LV and RV was performed from the conal part of the RV on the right side of interventricular septum corresponding to the area of the LV obstruction (3) (Copyrighted with permission of Oxford University Press). LV, left ventricle; RV, right ventricle; IVS, interventricular septum.

left ventricular cavity.

In 1993, Maron et al. (14) described a series of five patients with HCM and RV involvement. All had resting RV outflow gradients $>50 \mathrm{mmHg}$, with NYHA class 3 or 4 symptoms. Four of five patients also had significant LV outflow gradients at rest. In four patients, right ventriculotomy was performed, with substantial resection of hypertrophied RV tissue. In three of four HOCM patients with myotomy-myectomy, a right ventriculotomy was performed at the same operation. One patient died soon after the operation. Another one required mitral valve replacement two years after the operation. One other patient with simultaneous LVOT and RV obstruction had Morrow procedure alone. This patient died seven days after the surgery. In this report, all patients with right ventriculotomy had substantial portions of greatly hypertrophied RV muscle that were resected, including the crista and moderator band in two patients and the moderator band alone in one. These procedures are completely different from the surgical techniques that we have developed $(3,18)$. We have never resected the moderator band. Our experience showed $(3,16)$ that it was not necessary to use the resection for surgical correction of HOCM in patients with RV obstruction.

The optimal treatment for HOCM patients with significant RV disease, simultaneous RVOT and left ventricular outflow tract LVOT obstruction, and severe hypertrophy is unknown. In the absence of generally accepted standards for surgical correction of HOCM in patients with severe hypertrophy and biventricular obstruction, our technique of HOCM surgical correction was proposed $(3,16)$. Our surgical technique is based on excision of the hypertrophied tissue in the asymmetrical area of the IVS causing obstruction of LVOT, RVOT and left ventricular mid-cavity. Access to the hypertrophied area was achieved by entering through the conal part of the RV. A longitudinal incision in the conal part of the RV (length 20-28 mm) was made.

All attachments and additional trabecula between the anterior part of the ventricular septum and the RV anterior wall were divided. The excision of the hypertrophied area of the IVS causing obstruction was performed from the conal part of the RV corresponding to the zone obstruction of the LV (Figure 1B). This excision was carried out on the right side of the IVS, strictly anterior to the Lancisi muscle and below the pulmonary valve.

If the zone of IVS asymmetrical hypertrophy was more extensive than usual, the resection zone was extended upwards to the level of $2-3 \mathrm{~mm}$ below the base of the pulmonary trunk and downwards to the extension level. In all patients with simultaneous obstruction of left ventricular mid-cavity, RVOT, and LVOT, surgical excision of 

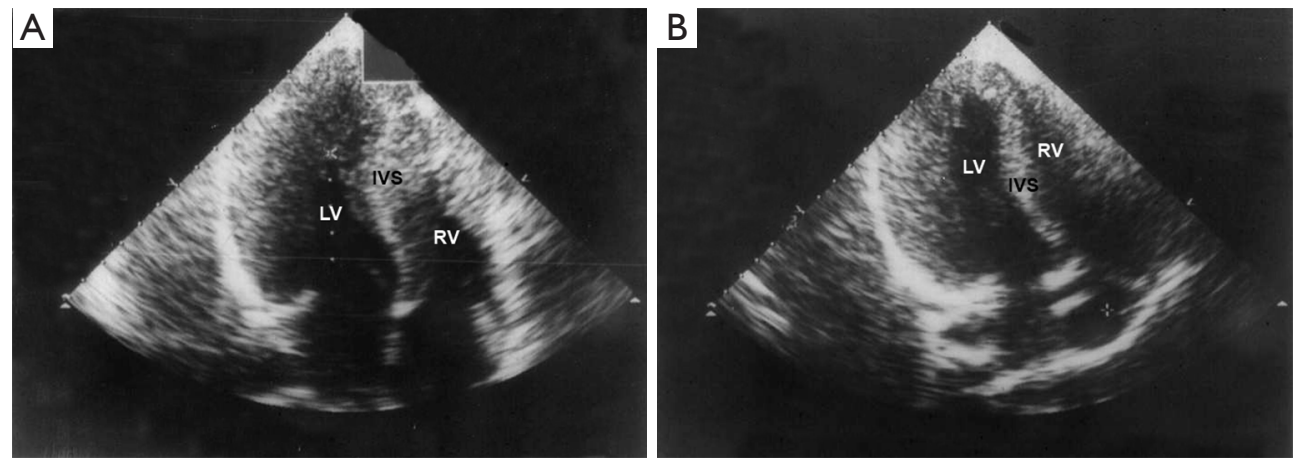

Figure 2 Preoperative (A) and postoperative (B) apical four chamber view in a 15 -year-old patient. (A) Four-chamber view with midventricular asymmetrical hypertrophy of the ventricular septum. (B) Four-chamber view showing a substantial decrease in the ventricular septum thickness in the middle part after surgery, an increase in the RV and LV cavity sizes. LV, left ventricle; RV, right ventricle; IVS, interventricular septum.

additional myocardium was also carried out only from the conal part of the $\mathrm{RV}$ and without penetration into the left ventricular cavity. Adequate resection was manifested by relaxation of the asymmetrical hypertrophy zone and was clearly defined by palpation. Excision of a third of the IVS on the right side at the level of asymmetrical hypertrophy was sufficient in most cases. Excision never exceeded half of IVS thickness.

Due to pressure difference in cavities of the left ventricles and $\mathrm{RV}$, smoothing of the relief of asymmetrical hypertrophy of the IVS left side occurs due to thinning of IVS, secondary to the described excision on the right side. It results in restoration of natural configuration of the left ventricle cavity.

In patients with severe $(>10 \mathrm{~mm})$ hypertrophy of the RV anterior wall, the partial excision of hypertrophied tissue in the area of maximal thickness of the anterior RV wall was made from the endocardial surface of the RV. The depth of this excision was approximately $3 \mathrm{~mm}$. Transesophageal echocardiographic evaluation focusing on the RV was performed intraoperatively.

\section{RV myectomy for surgical treatment of HOCM patients with biventricular obstruction}

A biventricular resection in HOCM patients is a highrisk procedure. The classic Morrow technique does not allow removal of the anatomical substrate of simultaneous obstruction of the left mid-ventricle and RVOT. We presented a series of seven patients with midventricular obstruction of the LV associated with RVOT obstruction [mean New York Heart Association (NYHA) class 3.0] that underwent our procedure (3). For patients with midventricular obstruction, the excision was made in the middle part of the right side of the IVS corresponding to the area of intraventricular obstruction of the LV. At followup to $24.8 \pm 11.3$ months, there were no early or late deaths or reoperations, six patients were free of symptoms (NYHA class 1 ), and one patient was in functional class 2 . The mean value of the echocardiographic intraventricular gradients in the $\mathrm{LV}$ decreased from $86.3 \pm 9.9$ to $10.3 \pm 5.3 \mathrm{mmHg}$, while gradients in the RVOT decreased to $44.9 \pm 9.6 v s$. $4.1 \pm 1.2 \mathrm{mmHg}$. The thickness of the ventricular septum was substantially reduced from $32.7 \pm 5.3$ to $15.6 \pm 3.2 \mathrm{~mm}$ (Figure 2). Sinus rhythm without right bundle branch block was noted in all patients after surgery. This surgical method therefore appears to provide an adequate resection of hypertrophic muscular tissue under direct visual control in both the upper and middle part of the IVS.

Heper et al. (19) presented a case of HOCM with LVOT and RVOT obstruction with surgical myectomy and graft patch interposition. Surgical treatment including an extensive muscular resection of the RVOT, minimal resection of the LVOT, and interposition of a graft patch in the RVOT resulted in disappearance of the RVOT gradient and a decrease to $28 \mathrm{mmHg}$ in the LVOT gradient. Alpat et al. (20) were concerned about using the patch to enlarge the RVOT during the operation. We never used patches for surgical correction of HOCM in patients with RVOT obstruction. For the purposes of widening of the RVOT and normalization of RV cavity size, it was sufficient to excise hypertrophic tissue in the asymmetrical area of the 
septum from the right side and divide additional trabecula and attachments between the anterior part of the ventricular septum and the RV anterior wall (21). The adequate resection of hypertrophied tissue from the right side resulted in elimination of obstruction, an increase in the RV and LV cavity sizes, and improvement in RV function.

\section{RV myectomy for surgical treatment of pediatric patients with $\mathrm{HOCM}$ and biventricular obstruction}

The need for heart transplantation in a pediatric patient several years after Morrow septal myectomy was reported, due to the development of severe biventricular outflow tract obstruction (22). Another study documented the death of a 26-year-old woman with a severe biventricular HCM after biventricular septal resection and left ventricular assist device implantation (23). Nine patients with severe obstruction of both RVOT and LVOT who underwent biventricular resection were excluded from the analysis of Minakata and coworkers (24). Another study (25) also showed that pediatric patients were excluded from the analysis because of concomitant RVOT obstruction.

Treatment of pediatric patients with HOCM is complicated by several factors (22). Major reasons include the difficulty for the surgeon to maintain sufficient visual control over the very narrow area to be resected, especially in young children, and the risk of septal perforation or damage to conductive tissue. The removal of myocardial tissue by the Morrow approach is near impossible if both left and RVOTs are obstructed.

In our study on $35 \mathrm{HOCM}$ pediatric patients with severe hypertrophy (16), all patients survived surgical correction. Preoperatively, 27 patients had biventricular obstruction and one patient had isolated RVOT obstruction. Surgical excision of additional myocardium was also performed from the conal part of the RV. During follow-up, ECG showed a newly induced right bundle branch block only in 1 of 35 patients. Children treated by this technique experienced a marked improvement in exercise tolerance and amelioration of dyspnea, chest pain, and presyncope. Furthermore, this study demonstrated significant reductions of LV intraventricular, LVOT, and RVOT gradients, as well as reductions of IVS thickness and left atrium size after surgical correction. Removal of obstructing tissue causing simultaneous obstruction of the left and right outflow tract, and avoidance of damage to the left side of the IVS and cardiac conduction system are important advantages of the surgical technique that we have applied.

In a study by Quintana et al. (15), relief of left ventricular obstruction was obtained by performing a transaortic extended septal myectomy and/or a left apical ventriculotomy in 11 symptomatic pediatric patients and young adults with biventricular outflow obstruction. RVOT obstruction was relieved with patch enlargement in all patients and selective resection of muscle bundles. If there were no chordal attachments at this level, a limited right septal myectomy was performed. There were no early deaths and two patients required permanent pacing for a complete heart block. At the last follow-up (median followup 4.6 years), 8 (72\%) patients were in functional class 1 .

In our cohort of HOCM patients, the excision of additional myocardium on the right side of the ventricular septum was performed strictly anterior to the Lancisi muscle and more close to the RV anterior wall. This conal region of the RV corresponds to the $\mathrm{LV}$ area of obstruction and the zone of the IVS asymmetrical hypertrophy. Using this approach, it is easy to avoid injury to the septal attachments of the tricuspid chordae tendinae.

\section{RV myectomy for HOCM patients with septal myocardial fibrosis}

In patients with HCM, myocardial fibrosis is an independent predictor of an adverse outcome $(26,27)$. The main cause of sudden death in HOCM is ventricular tachycardia/ fibrillation emanating from the areas of fibrosis (28).

For HOCM patients with septal myocardial fibrosis, the technique for RV myectomy has been modified. We presented a case report of a 33-year-old female with biventricular obstruction, extreme hypertrophy, septal myocardial fibrosis, and episodes of ventricular tachycardia, who underwent surgical correction according to this novel procedure (18). Septal myocardial fibrosis with areas of scarring in the basal and midventricular septum was detected by CMR with delayed enhancement (DE) imaging after gadolinium infusion.

The excision of the hypertrophied area of the interventricular septum causing simultaneous obstruction of the LV mid-cavity, RVOT, and LVOT was performed from the conal part of the $\mathrm{RV}$ on the right side of the IVS. The areas of septal myocardial fibrosis as identified by DE imaging were excised simultaneously under direct visual inspection in the middle part of the right side of the interventricular septum and in the upper third part. After surgery, NYHA functional class improved from 3 to 1 . 
The patient was free of symptoms (NYHA class 1) at the time of most recent contact (7 years after surgery). The LV intraventricular gradient remained low at $10 \mathrm{mmHg}$ and RVOT gradient at $3 \mathrm{mmHg}$. After surgery, thickness of ventricular septum was reduced from 33 to $18 \mathrm{~mm}$, RV cavity size was increased, and RV diastolic function was improved. The postoperative ECG showed a sinus rhythm without right and left bundle branch block. After surgical correction of HOCM and precise removal of septal myocardial fibrosis, the patient had no ventricular tachycardia and no syncopal or presyncopal episodes. The presented excision of the IVS allows one to avoid the negative consequences of damage to the right branch of the His bundle.

\section{RV myectomy for surgical treatment of HOCM patients after failed alcohol septal ablation}

Alcohol septal ablation (ASA) is ineffective in patients with substantial LV hypertrophy ( $>25 \mathrm{~mm}$ wall thickness), since sufficient septal thinning cannot be reliably achieved (29). Quintana et al. (30) observed a strong correlation between more advanced degrees of interstitial fibrosis and worsening diastolic function measured by strain at the septal level in patients after failed septal ablation. In addition to diastolic dysfunction, septal scarring from ASA may contribute to rhythm abnormalities. An earlier Mayo Clinic study also showed a ventricular tachyarrhythmia and complications rate of $20 \%$ with ASA (31).

Given the development of left bundle branch block in many HOCM patients after Morrow myectomy, patients who develop right bundle branch block after ASA have a higher likelihood of complete AV block after surgery (32). We proposed the technique of HOCM surgical correction without damage to the cardiac conduction system in patients with severe hypertrophy after unsuccessful ASA. Three symptomatic HOCM patients with obstruction in the left ventricular mid-cavity and severe septal hypertrophy (mean NYHA class 3) underwent surgical procedure at $14.0 \pm 7.0$ months after failed septal ablation. The excision was performed in the middle part of the right side of IVS corresponding to the area of $\mathrm{LV}$ intraventricular obstruction. After surgery, all three patients were free of symptoms (NYHA class 1). The mean echocardiographic intraventricular gradient in $\mathrm{LV}$ decreased from $78.0 \pm 8.0$ to $9.7 \pm 2.1 \mathrm{mmHg}$. Sinus rhythm was noted in all patients after surgery. No patients required pacemaker implantation. Tissue necrosis following ASA extended into the inferior portion of the septum at the midventricular level, primarily involving the RV portion (33). Our technique of HOCM surgical correction allows for the effective elimination of $\mathrm{LV}$ intraventricular obstruction in patients after unsuccessful ASA.

Limitations of published research include the small number of patients in currently available studies. Further investigation is needed to justify the selection of patients who may benefit from RV myectomy. A larger study population that includes HOCM patients with RV involvement and RV obstruction will be required.

\section{Conclusions}

HCM can involve the left and/or RV. The highest risk of sudden death was reported for patients with extreme hypertrophy (33 mm or thicker) $(34,35)$. The benefits of employing our surgical technique include: effective surgical treatment of HOCM patients with severe hypertrophy and biventricular obstruction, as well as the capacity for precise removal of areas of septal myocardial fibrosis. It is easier to remove fibrotic tissue from the right of the IVS side (corresponding to DE) under direct visual inspection. Our technique can be performed in symptomatic patients with severe obstructive HCM, providing excellent relief of symptoms. The removal of obstructing tissue causing simultaneous obstruction of the left mid-ventricle, RVOT and LVOT using the same approach and avoiding damage to the cardiac conduction system are important advantages of the surgical technique proposed.

This technique is indicated for the surgical treatment of HOCM patients with biventricular obstruction and for patients with isolated RVOT obstruction. It may be an appropriate choice for HOCM patients with severe hypertrophy and septal myocardial fibrosis. Future studies could further clarify the significance of RV myectomy for patients after failed alcohol septal ablation. Surgical RV myectomy may be a more prudent choice on the basis of $\mathrm{RV}$ outflow tract anatomy. These treatment options may offer novel solutions to a variable and complex HOCM population.

\section{Acknowledgements}

None.

\section{Footnote}

Conflicts of Interest: The author has no conflicts of interest to declare. 


\section{References}

1. Towbin J, Roberts R. Cardiovascular diseases due to genetic abnormalies. In: Schlant RC, Alexander RW. editors. Hurst's the Heart: Arteries and Veins, 8th ed. New York: McGraw-Hill Inc, 1994: 1725-59.

2. Morrow AG. Hypertrophic subaortic stenosis. Operative methods utilized to relieve left ventricular outflow obstruction. J Thorac Cardiovasc Surg 1978;76:423-30.

3. Borisov KV. Surgical correction of hypertrophic obstructive cardiomyopathy in patients with simultaneous obstruction of left ventricular midcavity and right ventricular outflow tract. Eur J Cardiothorac Surg 2013;43:67-72.

4. Teare D. Asymmetrical hypertrophy of the heart in young adults. Br Heart J 1958;20:1-8.

5. McKenna WJ, Kleinebenne A, Nihoyannopoulos P, et al. Echocardiographic measurement of right ventricle wall thickness in hypertrophic cardiomyopathy: relation to clinical and prognostic features. J Am Coll Cardiol 1988;11:351-8.

6. Fournier C, Bache R, Valette H, et al. Hypertrophic myocardiopathy with isolated obstruction of the right ventricle. Ann Cardiol Angeiol (Paris) 1985;34:71-4.

7. Maron MS, Hauser HH, Dubrow E, Horst TA, Kissinger $\mathrm{KV}$ et al. Right ventricular involvement in hypertrophic cardiomyopathy. Am J Cardiol 2007;100:1293-8.

8. Pagourelias ED, Efthimiadis GK, Parcharidou DG, et al. Prognostic value of right ventricular diastolic function indices in hypertrophic cardiomyopathy. Eur J Echocardiogr 2011;12: 809-17.

9. Finocchiaro G, Knowles JW, Pavlovich A, et al. Prevalence and clinical correlates of right ventricular dysfunction in patients with hypertrophic cardiomyopathy. Am J Cardiol 2014;113:361-7.

10. Nagata Y, Konno T, Fujino N, at al. Right ventricular hypertrophy is associated with cardiovascular events in hypertrophic cardiomyopathy: evidence from study with magnetic resonance imaging. Can J Cardiol 2015;31:702-8.

11. Lockhart A, Charpentier A, Bourdarias JP, et al. Right ventricular involvement in obstructive cardiomyopathies: hemodynamic studies in 13 cases. Brit Heart J 1966;28:122-33.

12. Casanova M, Gamallo C, Garcia-Aguado A, et al. Familial hypertrophic cardiomyopathy with unusual involvement of the right ventricle. Eur J Cardiol 1979:9:145-59.

13. Frank S, Braunwald E. Idiopathic hypertrophic subaortic stenosis. Clinical analysis of 126 patients with emphasis on the natural history. Circulation 1968;37:759-88.

14. Maron BJ, McIntosh CL, Klues HG, et al. Morphologic basis for obstruction to right ventricular outflow in hypertrophic cardiomyopathy. Am J Cardiol 1993;71:1089-94.

15. Quintana E, Johnson JN, Sabate-Rotes A, et al. Surgery for biventricular obstruction in hypertrophic cardiomyopathy in children and young adults: technique and outcomes. Eur J Cardiothorac Surg 2015;47:1006-12.

16. Borisov KV, Bockeria LA, Sinyov AF. Surgical treatment of hypertrophic obstructive cardiomyopathy in pediatric patients with severe hypertrophy. Artificial Organs 2008;32:856-63.

17. Dobell AR, Scott HJ. Hypertrophic subaortic stenosis: evolution of a surgical technique. Discussion. Harken DE. J Thorac Cardiovasc Surg 1964;47:26-39.

18. Borisov KV. Surgical correction of hypertrophic obstructive cardiomyopathy in a patient with severe hypertrophy and septal myocardial fibrosis. Interact Cardiovasc Thorac Surg 2012;15:769-71.

19. Heper G, Celiksoz M, Atasoy S, et al. Hypertrophic obstructive cardiomyopathy causing severe right and left ventricular outflow tract obstruction. Turk Kardiyol Dern Ars 2010;38:206-10.

20. Alpat S, Yilmaz M. Touch or keep away: big debate on mitral regurgitation in patients with hypertrophic obstructive cardiomyopathy. Eur J Cardiothorac Surg 2014;45:769.

21. Borisov KV. Reply to Alpat and Yilmaz. Eur J Cardiothorac Surg 2014;45:770.

22. Theodoro DA, Danielson GK, Feldt RH, et al. Hypertrophic obstructive cardiomyopathy in pediatric patients: results of surgical treatment. J Thorac Cardiovasc Surg 1996;112: 1589-97; discussion 1597-9.

23. McCully RB, Nishimura RA, Tajik AJ, et al. Extent of clinical improvement after surgical treatment of hypertrophic obstructive cardiomyopathy. Circulation 1996;94:467-71.

24. Minakata K, Dearani JA, O'Leary PW, et al. Septal myectomy for obstructive hypertrophic cardiomyopathy in pediatric patients: early and late results. Ann Thorac Surg 2005;80:1424-9; discussion 1429-30.

25. Stone CD, McIntosh CL, Hennein HA, et al. Operative treatment of pediatric obstructive hypertrophic cardiomyopathy: a 26-year experience. Ann Thorac Surg 1993;56:1308-13; discussion 1313-4.

26. O'Hanlon R, Grasso A, Roughton M, et al. Prognostic significance of myocardial fibrosis in hypertrophic 
cardiomyopathy. J Am Coll Cardiol 2010;56:867-74.

27. Adabag AS, Maron BJ, Appelbaum E, et al. Occurrence and frequency of arrhythmias in hypertrophic cardiomyopathy in relation to delayed enhancement on cardiovascular magnetic resonance. J Am Coll Cardiol 2008;51:1369-74.

28. Varnava AM, Elliott PM, Babboonian C, et al. Hypertrophic cardiomyopathy: histopathological features of sudden death in cardiac troponin $\mathrm{T}$ disease. Circulation 2001;104:1380-4.

29. Maron BJ, Nishimura RA. Surgical septal myectomy versus alcohol septal ablation. Assessing the status of the controversy in 2014. Circulation 2014;130:1617-24.

30. Quintana E, Sabate-Rotes A, Maleszewski JJ, et al. Septal myectomy after failed ablation: Does previous pecutaneous intervention compromise outcomes of myectomy? J Thorac Cardiovasc Surg 2015;150:159-67.e1.

31. Sorajja P, Valeti U, Nishimura RA, et al. Outcome of alcohol septal ablation for obstructive hypertrophic

Cite this article as: Borisov KV. Right ventricle myectomy. Ann Cardiothorac Surg 2017;6(4):402-409. doi: 10.21037/ acs.2017.07.10 cardiomyopathy. Circulation 2008;118:131-9.

32. Nagueh SF, Buergler JM, Quinones MA et al. Outcome of surgical myectomy after unsuccessful alcohol septal ablation for the treatment of patients with hypertrophic obstructive cardiomyopathy. J Am Coll Cardiol 2007;50:795-8.

33. Valeti US, Nishimura RA, Holmes DR, et al. Comparison of surgical sepatal myectomy and alcohol septal ablation with cardiac magnetic resonance imaging in patients with hypertrophic obstructive cardiomyopathy. J Am Coll Cardiol 2007;49:350-7.

34. Elliott PM, Gimeno Blanes J, Mahon NG, et al. Relation between severity of left-ventricular hypertrophy and prognosis in patients with hypertrophic cardiomyopathy. Lancet 2001;357:420-4.

35. Maron BJ, Piccininno M, Casey SA, et al. Relation of extreme left ventricular hypertrophy to age in hypertrophic cardiomyopathy. Am J Cardiol 2003;91:626-8. 